\title{
A case of Williams syndrome with suspected coexisting ectopic aldosterone-producing tumor in the liver
}

\author{
Shuhei Baba1, Arina Miyoshi ${ }^{1} 1$, Shinji Obara1, Hiroaki Usubuchi2, Satoshi Terae², Masao Sunahara³, \\ Takahiro Oshima3, Kazuhito Misawa3, Takahiro Tsuji4, Bunya Takahashi5, \\ Yuto Yamazaki ${ }^{6}$, Hironobu Sasano ${ }^{6}$ and Norio Wada ${ }^{1}$ \\ ${ }^{1}$ Departments of Diabetes and Endocrinology, ${ }^{2}$ Radiology, ${ }^{3}$ Surgery, 4 Pathology, Sapporo City General Hospital, \\ Sapporo, Japan, 5Department of Diagnostic Imaging, Faculty of Medicine and Graduate School of Medicine, Hokkaido \\ University, Sapporo, Japan, and ${ }^{6}$ Department of Pathology, Tohoku University Graduate School of Medicine, \\ Sendai, Japan
}

Correspondence should be addressed to $\mathrm{N}$ Wada

Email

norio.wada@doc.city. sapporo.jp

\section{Summary}

A 31-year-old man with Williams syndrome (WS) was referred to our hospital because of a 9-year history of hypertension, hypokalemia, and high plasma aldosterone concentration to renin activity ratio. A diagnosis of primary aldosteronism (PA) was clinically confirmed but an abdominal CT scan showed no abnormal findings in his adrenal glands. However, a 13-mm hypervascular tumor in the posterosuperior segment of the right hepatic lobe was detected. Adrenal venous sampling (AVS) subsequently revealed the presence of an extended tributary of the right adrenal vein to the liver surrounding the tumor. Segmental AVS further demonstrated a high plasma aldosterone concentration (PAC) in the right superior tributary vein draining the tumor. Laparoscopic partial hepatectomy was performed. The resected tumor histologically separated from the liver was composed of clear cells, immunohistochemically positive for aldesterone synthase (CYP11B2), and subsequently diagnosed as aldosterone-producing adrenal adenoma. After surgery, his blood pressure, serum potassium level, plasma renin activity and PAC were normalized. To the best of our knowledge, this is the first report of WS associated with PA. WS harbors a high prevalence of hypertension and therefore PA should be considered when managing the patients with WS and hypertension. In this case, the CT findings alone could not differentiate the adrenal rest tumor. Our case, therefore, highlights the usefulness of segmental AVS to distinguish adrenal tumors from hepatic adrenal rest tumors.

\section{Learning points:}

- Williams syndrome (WS) is a rare genetic disorder, characterized by a constellation of medical and cognitive findings, with a hallmark feature of generalized arteriopathy presenting as stenoses of elastic arteries and hypertension.

- WS is a disease with a high frequency of hypertension but the renin-aldosterone system in WS cases has not been studied at all.

- If a patient with WS had hypertension and severe hypokalemia, low PRA and high ARR, the coexistence of primary aldosteronism (PA) should be considered.

- Adrenal rest tumors are thought to arise from aberrant adrenal tissues and are a rare cause of PA.

- Hepatic adrenal rest tumor (HART) should be considered in the differential diagnosis when detecting a mass in the right hepatic lobe.

- Segmental adrenal venous sampling could contribute to distinguish adrenal tumors from HART. 

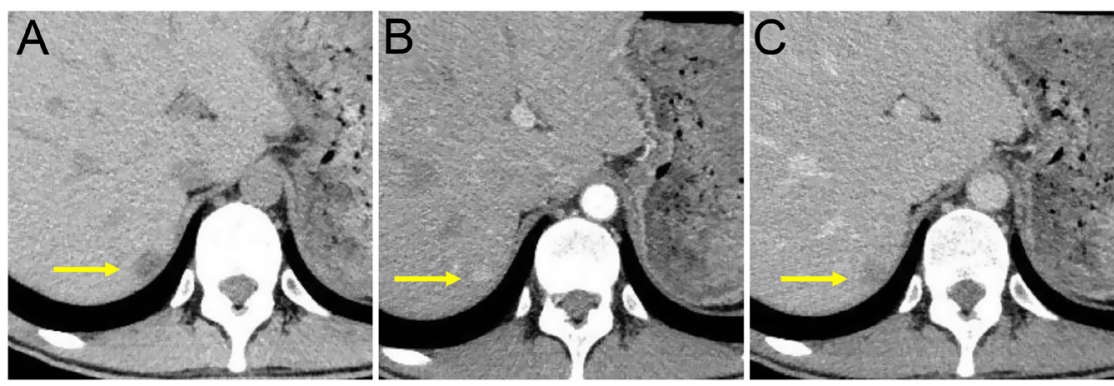

\section{Figure 1}

Enhanced abdominal CT of the initial visit. (A) Pre-contrast, (B) arterial phase, and (C) venous phase. A 13-mm hypodense mass shown in the posterosuperior segment of the right hepatic lobe (arrow) that appeared to be embedded within the liver. The mass is enhanced in the arterial phase and washed out in the portal phase.

\section{Background}

Williams syndrome (WS) is a microdeletion disorder caused by heterozygous loss of approximately $1.5 \mathrm{Mbp}$ of DNA from chromosome 7, including the elastin gene, which codes for the protein elastin. Patients with WS have a characteristic constellation of medical and cognitive findings, with a hallmark feature of generalized arteriopathy presenting as stenoses of elastic arteries and hypertension (1). Since the first report of Daniels et al. (2), the causal relationship between arterial stenosis and hypertension has been demonstrated; however, few reports are available on the relationship with endocrine diseases, including primary aldosteronism (PA), which is the most common cause of endocrinerelated hypertension (3). Here, we present a case of WS associated with PA whose source of aldosterone excess was difficult to locate.

\section{Case presentation}

A 31-year-old man was admitted for further evaluation of clinically suspected PA. He was diagnosed as WS at 2 months of age by genetic analysis. He had clinically mild cardiovascular complications such as mild supravalvular aortic stenosis diagnosed at 2 months and also mild peripheral pulmonic stenosis detected at 15 years of age. He had been hypertensive for 9 years. On admission, his blood pressure was 146/93 $\mathrm{mmHg}$ while taking amlodipine $10 \mathrm{mg}$ daily. His height and weight were $158 \mathrm{~cm}$

Table 1 Results of captopril challenge test.

\begin{tabular}{|c|c|c|c|}
\hline & \multicolumn{3}{|c|}{ Times (min) } \\
\hline & 0 & 60 & 90 \\
\hline PRA (ng/mL/h) & $<0.2$ & $<0.2$ & $<0.2$ \\
\hline PAC (pg/mL) & 522 & 465 & 589 \\
\hline ARR & 2610 & 2280 & 2945 \\
\hline
\end{tabular}

ARR, aldosterone to renin ratio; PAC, plasma aldosterone concentration; PRA, plasma renin activity. and $49 \mathrm{~kg}$, respectively. He had no notable family history. On physical examination, he had dysmorphic features associated with WS, such as the typical elfin face (broad forehead, periorbital fullness, a flat nasal bridge with an upturned nose, long philtrum, and full lips). He had a systolic murmur (Grade 2/4) at the base of the heart.

\section{Investigation}

Routine laboratory tests revealed a low potassium level of $3.1 \mathrm{mEq} / \mathrm{L}$ (normal: $3.5-5.0 \mathrm{mEq} / \mathrm{L}$ ) despite taking potassium chloride of $24 \mathrm{mEq} /$ day. Urinalysis results were noncontributory. A captopril challenge test (CCT) confirmed the diagnosis of PA (Table 1). A CT scan showed no abnormal findings in his adrenal glands, but a 13-mm hypodense and hypervascular tumor in the posterosuperior segment of the right hepatic lobe (Segment 7) was detected (Fig. 1A, B, and C). He was hospitalized for further investigation. Endocrinological data showed that the plasma aldosterone concentration (PAC) was $451 \mathrm{pg} / \mathrm{mL}$, and the plasma renin activity (PRA) was less than $0.2 \mathrm{ng} / \mathrm{mL} / \mathrm{h}$ (taken at 08:00 h). The aldosterone to renin ratio (ARR) was calculated 2255 . Serum adrenocorticotropic hormone (ACTH), cortisol, and catecholamine levels were within the normal
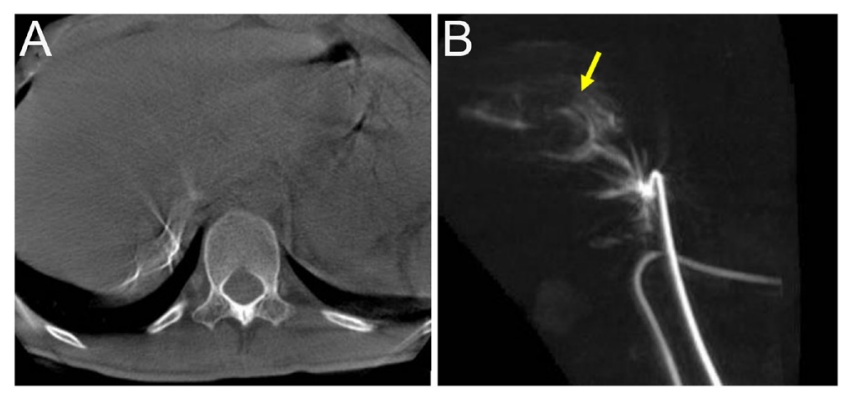

\section{Figure 2}

Venography at adrenal venous sampling (AVS) (A) transverse and (B) coronal views. Intraprocedural real-time cone-beam CT imaging reveals an extended tributary of the right adrenal vein to the liver that surrounded the tumor (allows). 
Table 2 Results of ACTH-stimulated adrenal venous sampling.

\begin{tabular}{|c|c|c|c|c|c|}
\hline & $\begin{array}{c}\text { Cortisol } \\
(\mu \mathrm{g} / \mathrm{dL})\end{array}$ & $\begin{array}{c}\text { Aldosterone } \\
(\mathrm{pg} / \mathrm{mL})\end{array}$ & $A / C$ & LR & CR \\
\hline \multicolumn{6}{|c|}{$\overline{\text { Before ACTH stimulation }}$} \\
\hline IVC & 16.8 & 786 & 46.8 & & \\
\hline Right AV & 26.2 & 3780 & 114.3 & 15.9 & \\
\hline Left AV & 285 & 2040 & 7.2 & & 0.15 \\
\hline \multicolumn{6}{|c|}{ After ACTH stimulation } \\
\hline IVC & 18.1 & 695 & 38.4 & & \\
\hline Right AV & 648 & 26500 & 40.9 & 4.98 & \\
\hline Left AV & 722 & 5910 & 8.2 & & 0.22 \\
\hline
\end{tabular}

$\mathrm{A} / \mathrm{C}$, aldosterone-cortisol ratio; $\mathrm{AV}$, adrenal vein; $\mathrm{ACTH}$,

adrenocorticotropic hormone; CR, contralateral ratio; IVC, inferior vena cava; LR, lateralized ratio.

range. A 1-mg overnight dexamethasone suppression test showed serum cortisol suppression $(<0.2 \mu \mathrm{g} / \mathrm{dL})$. Adrenal venous sampling (AVS) was then performed. After ACTH stimulation, the PAC, lateralized ratio (LR), and contralateral ratio (CR) were $26500 \mathrm{pg} / \mathrm{mL}$, 4.98 , and 0.22 , respectively, suggesting right-sided aldosterone hypersecretion. However, imaging did not reveal an abnormality on the right adrenal gland (Table 2). Besides, remarkably, on venography during AVS, an extended tributary of the right adrenal vein to the liver that surrounded the tumor (Fig. 2A and B). Thus, right segmental AVS (S-AVS) in the right adrenal vein was added and demonstrated an extremely high PAC (37 $700 \mathrm{pg} / \mathrm{mL})$ and PAC/PCC ratio (3459) in the superior tributary vein drained from the tumor (Fig. 3 and Table 3). In contrast, PAC obtained from the other veins was suppressed. On MRI, the opposed-phase T1-weighted sequence revealed dropout at the lesion of segment 7 , which indicated lipid content. On Gd-EOB-DTPA-enhanced MRI, the lesion was enhanced in the arterial phase and had low intensity in the hepatobiliary phase. An I-131-labeled adosterol scintigraphy revealed significant uptake in the bilateral adrenal glands and the tumor. In addition, the accumulation of the right adrenal gland and the tumor seemed to be inseparable from one another.
Table 3 Results of segmental adrenal venous sampling for right adrenal vein.

\begin{tabular}{|c|c|c|c|}
\hline & \multicolumn{3}{|c|}{ Tributary } \\
\hline & Superior & Lateral & Inferior \\
\hline Aldosterone (pg/mL) & 377000 & 18800 & 4450 \\
\hline Cortisol ( $\mu \mathrm{g} / \mathrm{dL})$ & 109 & 648 & 521 \\
\hline $\mathrm{A} / \mathrm{C}$ & 3458.7 & 29.0 & 8.54 \\
\hline
\end{tabular}

$\mathrm{A} / \mathrm{C}$, aldosterone-cortisol ratio

\section{Treatment}

Based on the findings described previously, PA caused by the tumor located in segment 7 of the liver was suspected. HCC with massive fatty metamorphosis was not completely ruled out and laparoscopic partial hepatectomy was subsequently performed.

\section{Clinical outcome and follow-up}

The resected tumor appeared golden yellow on the cut surface and macroscopically separated from the adjacent liver by a fibrous septum (Fig. 4A). Microscopic findings revealed that the tumor was connected to the adrenal cortex attached to the liver (Fig. 4B and C). The tumor was histologically composed of clear cells, immunohistochemically positive for aldosterone synthase (CYP11B2), which was diagnosed as an aldosteroneproducing adrenal adenoma (Fig. 4E). At 1 year after surgery, his blood pressure was normalized without oral medication and his serum potassium level, PRA, and PAC were normalized.

\section{Discussion}

To the best of our knowledge, this is the first report of WS associated with PA. Individuals with WS present with a highly characteristic phenotypic profile but the prevalence of systemic hypertension is a common finding with WS. The literature review revealed that the prevalence of
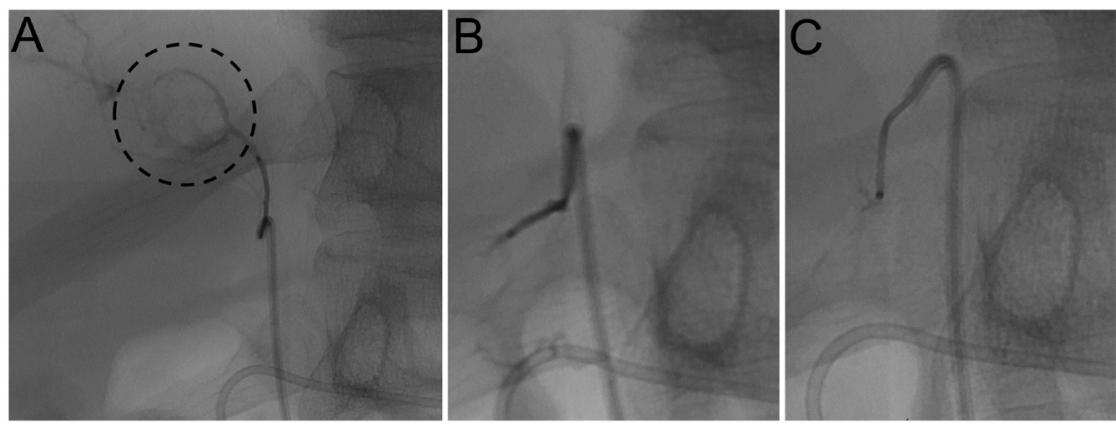

Figure 3

Fluoroscopic images of segmental adrenal venous sampling (S-AVS) for right adrenal vein. (A) Coronal views of the superior tributary vein, (B) lateral tributary vein, and (C) inferior tributary vein. 

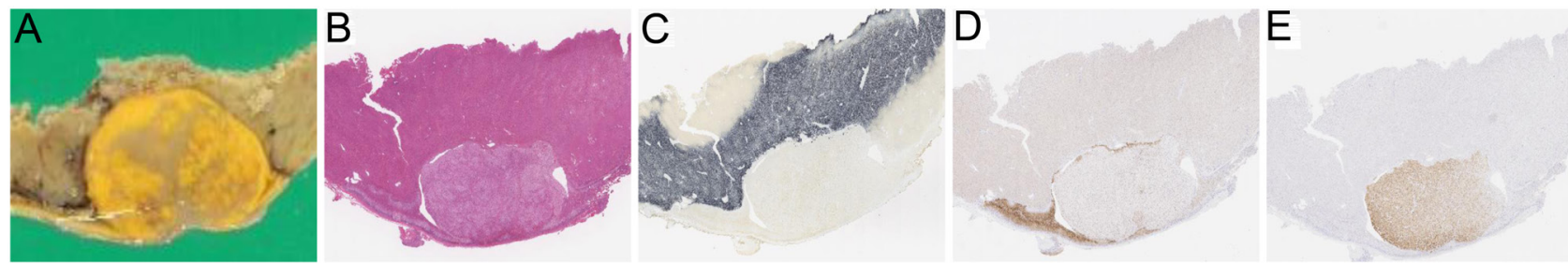

Figure 4

(A) Macroscopic appearance of the resected tumor, (B) hematoxylin and eosin staining, (C) immunohistochemistry of nuclear receptor 5A1 (steroidogenic factor 1) and immunohistochemistry using hepatocyte paraffin 1, (D) immunohistochemistry of 11-beta-hydroxylase (CYP11B1 and (E)

immunohistochemistry of aldosterone synthase (CYP11B2).

hypertension in WS varied from 5 to $70 \%$ in different series $(4,5,6)$, and there is a lifetime risk of developing arterial hypertension of $50 \%$ (5). The mean age at diagnosis of hypertension varied widely in the literature, from 4.7 to 38.8 years $(4,6,7)$, and hypertension could develop during infancy. Heterozygous microdeletion of the elastin gene is well-known to lead to vascular stenosis and appears to predispose to hypertension but the etiology has been clarified only in a small minority of the patients with the overt renovascular disease and classified as idiopathic in the great majority of the patients $(6,7)$. WS is frequently associated with hypertension but its renin-aldosterone system has remained largely unknown. Hypertension in WS is generally attributed to vascular stenosis, while the possibility of a certain number of PAs cannot be ruled out. In our case, severe hypokalemia, low PRA and high ARR raised the suspicion of the accidental coexistence of PA. Therefore, WS and PA are thought to coexist accidentally. If a patient of WS had hypertension with severe hypokalemia, measurement of PRA and PAC is recommended.

In this case, based on the CT findings, an ectopic PA arising from a hepatic adrenal rest tumor (HART) was clinically suspected because the tumor appeared to be embedded in the liver and separated from the right adrenal gland. Adrenal rest tumor is derived from ectopic adrenocortical tissue and has been reported to occur most commonly in abdominal and pelvic sites but rarely reported in the liver (8). Radiologically, HART is typically a round and well-defined mass in the right hepatic lobe, containing fat components, and is supplied mainly by the hepatic artery $(9,10)$. However, it is also true that these features could be also detected in other hepatic tumors, including HCC, which makes accurate preoperative radiological diagnosis extremely difficult. The demonstration of excess steroid hormone production may be helpful to discriminate but the great majority of HART reported has been clinically indolent. In our present case, pathological findings demonstrated that an adrenal adenoma originated from the right adrenal cortex rather than being attached to the liver. If HART could possibly produce and secrete excessive amounts of aldosterone, a step-up of aldosterone concentration in adrenal vein sampling should not occur. Therefore, segmental AVS is useful for distinguishing adrenal tumor from HART.

\section{Declaration of interest}

The authors declare that there is no conflict of interest that could be perceived as prejudicing the impartiality of this case report.

\section{Funding}

This research did not receive any specific grant from any funding agency in the public, commercial, or not-for-profit sector.

\section{Patient consent}

Written informed consent was obtained from the patient for publication of this case report and the accompanying images.

\section{Author contribution statement}

Shuhei Baba wrote the report and performed a literature review. Arina Miyoshi and Shinji Obara contributed to patient care as part of the Department of Diabetes and Endocrinology. Hiroaki Usubuchi, Satoshi Terae, and Bunya Takahashi provided radiological diagnostic support and performed adrenal venous sampling. Kazuhito Misawa performed partial hepatectomy. Takahiro Tsuji, Yuto Yamazaki, and Hironobu Sasano performed pathological examinations. Norio Wada oversaw the creation of the report and managed the patient's endocrine and general medical care. All the authors approved the final version for publication.

\section{References}

1 Williams JC, Barratt-Boyes BG \& Lowe JB. Supravalvular aortic stenosis. Circulation 196124 1311-1318. (https://doi.org/10.1161/01. cir.24.6.1311)

2 Daniels SR, Loggie JM, Schwarts DC, Strife JL \& Kaplan S. Systemic hypertension secondary to peripheral vascular anomalies in patients with Williams syndrome. Journal of Pediatrics 1985106 249-251. (https://doi.org/10.1016/s0022-3476(85)80297-3)

3 Omura M, Saito J, Yamaguchi K, Kakuta Y \& Nishikawa T. Prospective study on the prevalence of secondary hypertension among hypertensive 
patients visiting a general outpatient clinic in Japan. Hypertension Research 200427 193-202. (https://doi.org/10.1291/hypres.27.193)

4 Pober BR, Johnson M \& Urban Z. Mechanism and treatment of cardiovascular disease in Williams-Beuren syndrome. Journal of Clinical Investigation 2008118 1606-1615. (https://doi.org/10.1172/JCI35309)

5 Del Campo M, Antonell A, Magano LF, Munoz FJ, Flores R, Bayes M \& Perez Jurado LA. Hemizygosity at the NCF1 gene in patients with Williams-Beuren syndrome decreases their risk of hypertension. American Journal of Human Genetics 200678 533-542. (https://doi. org/10.1086/501073)

6 Bouchireb K, Boyer O, Bonnet D, Brunelle F, Decramer S, Landthaler G, Liutkus A, Niaudet P \& Salomon R. Clinical features and management of arterial hypertension in children with WilliamsBeuren syndrome. Nephrology, Dialysis, Transplantation 201025 434-438. (https://doi.org/10.1093/ndt/gfp522)
7 Furusawa EA, Esposito CSL, Honjo RS, Suzuki L, Leal GN, Kim CA \& Schvartsman BGS. Diagnosis and management of systemic hypertension due to renovascular and aortic stenosis in patients with Williams-Beuren syndrome. Revista da Associação Médica Brasileira 201864 723-728. (https://doi.org/10.1590/1806-9282.64.08.723)

8 Schechter DC. Aberrant adrenal tissue. Annals of Surgery 1968167 421-426. (https://doi.org/10.1097/00000658-196803000-00017)

9 Shin YM. Hepatic adrenal rest tumor mimicking hepatocellular carcinoma. Korean Journal of Hepatology 201016 338-341. (https:// doi.org/10.3350/kjhep.2010.16.3.338)

10 Tajima T, Funakoshi A, Ikeda Y, Hachitanda Y, Yamaguchi M, Yokota M, Yabuuchi H, Satoh T \& Koga M. Nonfunctioning adrenal rest tumor of the liver: radiologic appearance. Journal of Computer Assisted Tomography 200125 98-101. (https://doi. org/10.1097/00004728-200101000-00018)

Received in final form 17 June 2020

Accepted 25 August 2020 